\title{
2-D Optical-CDMA Modulation in Automotive Time-of-Flight LIDAR Systems
}

\author{
Wing C. Kwong, Senior Member, IEEE, Wei-Yi Lin*, Guu-Chang Yang*, Fellow, IEEE, \\ and Ivan Glesk**, Senior Member, IEEE \\ Department of Engineering, Hofstra University, Hempstead, USA \\ * Department of Electrical Engineering and Graduate Institute of Communication Engineering, \\ National Chung Hsing University, Taichung 402, Taiwan \\ ** Faculty of Engineering, University of Strathclyde, Glasgow G1 1XW, UK \\ Tel: +1 (516) 463 6564, Fax: +1 (516) 463 4939, e-mail: wing.c.kwong@hofstra.edu
}

\begin{abstract}
In this paper, the application of 2-D optical code-division multiple-access (OCDMA) modulation using wavelength-hopping time-spreading codes in long-range automotive time-of-flight (ToF) light detection and ranging (LIDAR) systems is proposed and demonstrated. The regulations and constraints that govern the design of the proposed system are presented. Using 2-D carrier-hopping prime codes, the modulation technique and associated hardware are implemented and validated with OptiSystem ${ }^{\mathrm{TM}}$ simulation. Interference-robustness studies show that the proposed 2-D OCDMA ToF LIDAR system is more flexible in the choice of system/code parameters and can support many more sensors and simultaneous ToF measurements than the 1-D counterpart.
\end{abstract}

Keywords: Automotive, code division multiple access, LIDAR, time-of-flight.

\section{INTRODUCTION}

The long range, pulse scanning, time-of-flight (ToF) measurement is one promising application of automotive light detection and ranging (LIDAR) systems [1]-[3]. A ToF signal consists of a train of narrow laser pulses transmitted periodically from a sensor of a LIDAR-equipped vehicle. The round-trip time of these pulses reflected from an object is used to calculate the sensor-object distance. Recent studies showed that the use of optical codedivision multiple-access (OCDMA) modulation with 1-D time-spreading sequences and avalanche photodetectors (APDs) could support such long range, laser safe, ToF measurement without complex signal processing [2], [3]. As every ToF laser pulse was encoded into a (pseudo-orthogonal) time-spreading sequence (e.g., optical orthogonal codes (OOCs) and prime codes [4]-[7]), these sequences could serve as distinctive tags of ToF signals and thus support sensor identification to reduce range ambiguity and false detections. The 1-D OCDMA modulation could extend the ToF distance and support faster scanning rate and better angular resolution than the classical single-pulse method [2], [3]. However, Fersch, et al. [2] focused on the correlation properties of OOCs when they were not overlapping in time. This was basically a single-sensor study and the interference robustness of the 1-D OCDMA modulation was not demonstrated. If multiple sequences (i.e., ToF signals) can be distinguished at the receiver of a sensor, multiple simultaneous ToF measurements can be supported, thus resulting in even faster scanning/frame rate and better angular resolution. Moreover, due to the stringent restriction in the code parameters of the time-spreading sequences [6], [7], the 1-D OCDMA modulation can support very few sensors after laser-safety regulations and hardware constraints have been taken into consideration [2], [3].

This paper addresses the above deficiencies by applying 2-D wavelength-hopping time-spreading (or, in short, wavelength-time) codes [6]-[9]. Section 2 studies the proposed 2-D CDMA modulation in the fast-scanning, longrange, APD-based, automotive ToF LIDAR system. For illustration, each sensor is assigned a unique sequence from the 2-D carrier-hopping prime codes (CHPCs) [6], [7], which have the advantages of enlarged cardinality by decoupling code weight from code length [8], [9] and very low cross-correlation function. Hence, the 2-D CHPCs can support more sensors and heavier code weight than 1-D time-spreading codes, thus enhancing interference robustness, making false detections less likely, and supporting many more simultaneous ToF measurements for faster scanning/frame rate and better angular resolution. Also, the restrictions caused by laser-safety regulations and hardware constraints in the design of the proposed system are investigated. Section 3 reports the OptiSystem ${ }^{\mathrm{TM}}$ simulation set-up of the proposed 2-D OCDMA automotive ToF LIDAR system. The interference-robustness studies in Section 4 show that the proposed 2-D system is more flexible in the choice of system/code parameters and can support many more sensors and simultaneous ToF measurements than the 1-D counterpart.

\section{AUTOMOTIVE TOF LIDAR SYSTEM AND DESIGN CONSTRAINTS}

Figure 1a shows the block diagram of the proposed 2-D OCDMA automotive ToF LIDAR sensor module. At the transmitting side, a laser diode is used to generate a narrow optical pulse in a fixed interval (i.e., repetition period). The pulse width, power, and period are governed by various factors, such as laser-safety regulations, hardware speed, number of measurement points, and angular resolution [1]-[3]. Each laser pulse is encoded into a code sequence in the 2-D OCDMA encoder. (The encoder's configuration is shown in Section 3.) A scanner is used to 
direct the code sequence (i.e., ToF signal) to a targeted object. For steering the ToF signal to perform pixel-bypixel measurement, the scanner can be made of microelectromechanical systems (MEMS) scanning mirror or a rotating mirror [2], [3]. At the receiving side, multiple code sequences will arrive and are detected by the APD simultaneously. While some of them can be originated from the same sensor but at adjacent measurement points, some can come from the reflection of different objects or from different sensors. The 2-D OCDMA decoder is configured as an inverted filter of its associated encoder with a reversed code sequence as its address signature. An arrival sequence with the correct address (i.e., from the transmitter of the same sensor) will create an autocorrelation peak at the decoder, while incorrect sequences (i.e., interference) will create a cross-correlation function [5]-[9]. A pulse demodulator threshold-detects the autocorrelation peak and restores the proper format of the TOF signal. Finally, a timer records the round-trip time of the ToF signal between the sensor and object.

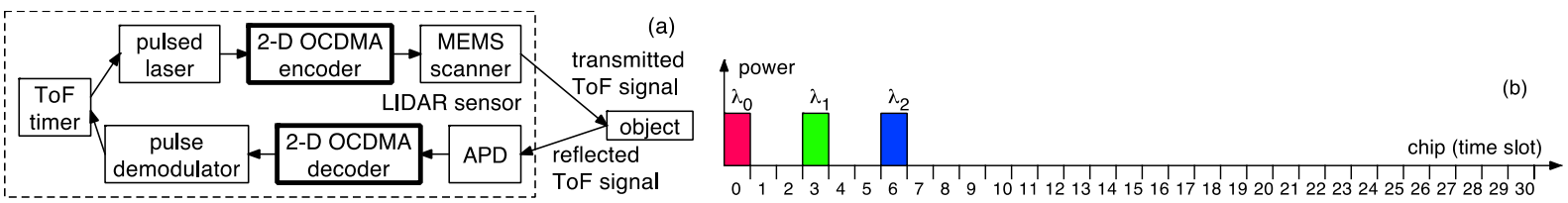

Figure 1. (a) Block diagram of the proposed 2-D CDMA automotive ToF LIDAR sensor module. (b) 2-D

CHPC sequence $X_{3}=(0,3,6)$ of weight $w=3, L=3$ wavelengths, and $N=31$ chips. Each color indicates one wavelength $(\lambda)$ of a laser pulse in the CHPC sequence.

The CHPCs belong to a family of 2-D asynchronous wavelength-time codes of $L$ wavelengths, $N$ chips (i.e., length), $w$ pulses (i.e., weight), and cardinality of $N$, where $w \leq L \leq N$ [6], [7]. Using $N=31$ and $w=L=3$ as an example, there exist 31 CHPC sequences of 3 wavelengths and 31 chips, denoted as $X_{0}=(0,0,0), X_{1}=(0,1,2)$, $X_{2}=(0,2,4), X_{3}=(0,3,6), X_{4}=(0,4,8), \ldots$, and $X_{30}=(0,30,29)$, where the numbers represent the chip positions of the three wavelengths. As shown in Figure $1 \mathrm{~b}, X_{3}=(0,3,6)$ means that the three wavelengths $\lambda_{0}, \lambda_{1}$, and $\lambda_{2}$ are used at chips 0,3 , and 6, respectively. (Details of the construction algorithm can be found in [6] and [7].)

To apply OCDMA modulation in the ToF LIDAR measurement, the system and code parameters are governed by laser-safety regulations and hardware constraints. To be safe to human eyes, the admissible exposure limit per pixel (AEL $L_{\text {pixel }}$ ) restricts the maximum optical energy within each measurement period. Hence, the optical power of each of the $w$ pulses (of width $\tau$ ) in the code sequences is restricted to $P_{\text {chip }}=\mathrm{AEL}$ pixel $/(\tau w)$ [2], [3]. The smaller the number of pulses $w$ or narrower the chip-width $\tau$, the higher the pulse power becomes. As the intensity of the received pulses is inversely proportional to the square of the ToF distance, higher pulse power used for transmission and, in turn, seen at the receiver can support longer distance measurement due to higher signal-tonoise (power) ratio against background and hardware noises. In the ToF LIDAR systems, the maximum measurement distance is usually given priority over accuracy or precision. Kim and Park [3] showed that it was best to use smaller number of pulses (e.g., $w \approx 3$ ) to lengthen the ToF measurement distance.

Besides code weight, there is a restriction on code length due to laser-beam steering in the MEMS or rotating mirrors. For long code length and high scanning (i.e., lateral movement) speed, the laser beam will already be moved to a different spot at the target, thus resulting in blurry or incomplete ToF measurement [2]. Hence, the code length will need to be adjusted in accordance to the scanning speed. Due to the scanner's constraints and long-range use, a sensor-object distance of at least $D=100 \mathrm{~m}$ is assumed. The ToF distance measurement thus requires a repetition period of at least $2 D / c=666.7 \mathrm{~ns}$, where $c=3 \times 10^{8} \mathrm{~m} / \mathrm{s}$ is the speed of light. Assume that the scanner scans over a horizontal field-of-view of $\alpha=60^{\circ}$ in $T_{\text {hor }}=125 \mu$ s (a reasonable value for the MEMS mirrors) for $D=100 \mathrm{~m}$ [2]. The lateral movement of the laser beam becomes $\mathrm{D} \times \tan \left(\mathrm{N} \tau \alpha / T_{\text {hor }}\right)=0.13 \mathrm{~m}$ (acceptable as a complete ToF measurement at a spot) when code length $N=31$ and chip-width $\tau=5$ ns are used. For $N=31$ and $\tau=5 \mathrm{~ns}$, the scanning or measurement period needs to be at least $31 \times 5=155 \mathrm{~ns}$, which is within the required period of $666.7 \mathrm{~ns}$. Hence, as used in [2] and [3], $N=31$ is about the largest allowable code length for the OCDMA ToF LIDAR system so that the whole sequence can be assumed to hit the same spot.

The code parameters and correlation properties of the 1-D and 2-D sequences impose a restriction to the cardinality, which is governed by the Johnson's cardinality upper bound [6], [7]

$$
\Phi \leq \frac{L(L N-1)(L N-2) \cdots\left(L N-\lambda_{C}\right) \lambda_{a}}{w(w-1)(w-2) \cdots\left(w-\lambda_{c}\right)}
$$

where $\lambda_{c}>0$ is the maximum cross-correlation value and $\lambda_{a}>0$ is the maximum autocorrelation sidelobe value of the code sequences in use. A large code weight $w$ can enhance interference robustness of ToF signals because of high autocorrelation peak. However, a large $w$ limits the number of supported sensors due to (1) and diminishes the ToF measurement range due to lower peak power, as explained earlier. As it is more important to have a long measurement range than accuracy or precision, $w \approx 3$ was used [2], [3]. Also, $N \approx 31$ is required because of the scanning-rate and angular-resolution requirements. As a result, the cardinality upper bound of 1-D time-spreading sequences of $w=3, N=31, L=1$ (i.e., one wavelength), and $\lambda_{c}=\lambda_{a}=1$ is limited to $\Phi=(1 \times 31-1) /[3 \times(3-1)]=5$ 
in (1). In fact, the 1-D OOCs of $w=3$ and $N=31$ used in [2] and [3] have 5 sequences, meaning that only up to 5 sensors can be supported. As shown in Section 4, the number of active sensors is even smaller.

The cardinality limit is relaxed in 2-D wavelength-time codes because the weight $w$ and length $N$ are decoupled and the cardinality can be enlarged by increasing the number of wavelengths or length [8], [9]. For example, the 2-D CHPCs of $N=31$ can support 31 sequences (i.e., sensors) and $w$ can be any integer up to $N$. Because of using multiple wavelengths, 2-D wavelength-time codes can support many more sensors and simultaneous ToF measurements with stronger interference robustness than 1-D time-spreading codes, as shown in Section 4.

\section{2-D OCDMA TOF LIDAR SYSTEM SIMULATION MODEL}

OptiSystem $^{\mathrm{TM}}$ is used to simulate the proposed 2-D OCDMA ToF LIDAR system. Figure 2 shows the simulation set-up of the transmitter in a sensor module. A bit sequence generator is used to repeatedly trigger an NRZ pulse generator to create a pulse of width $\tau=5$ ns (i.e., chip-width) for the 2-D tuneable encoder to generate a CHPC sequence of length $N=31$ at a repetition period of 155 ns. For weight $w=3$ and $L=3$ wavelengths, the encoder consists of a $1 \times 3$ splitter, which split every incoming pulse into 3 pulses, one for each wavelength path. To create the CHPC sequence $X_{3}=(0,3,6)$, the delay-lines on the middle and bottom paths are programmed to create 3 and 6 chips (i.e., 15 and $30 \mathrm{~ns}$ ) of time delays, respectively. The three delayed pulses then trigger their direct-modulated lasers of wavelengths $\lambda_{0}=650, \lambda_{1}=660$, and $\lambda_{2}=670 \mathrm{~nm}$, respectively. Finally, the three laser pulses are recombined at a wavelength multiplexer (Mux) to form $X_{3}$, which is then transmitted as a ToF signal. By programming the delay-lines accordingly, every one of the 31 CHPC sequences of $N=31$ can be generated.

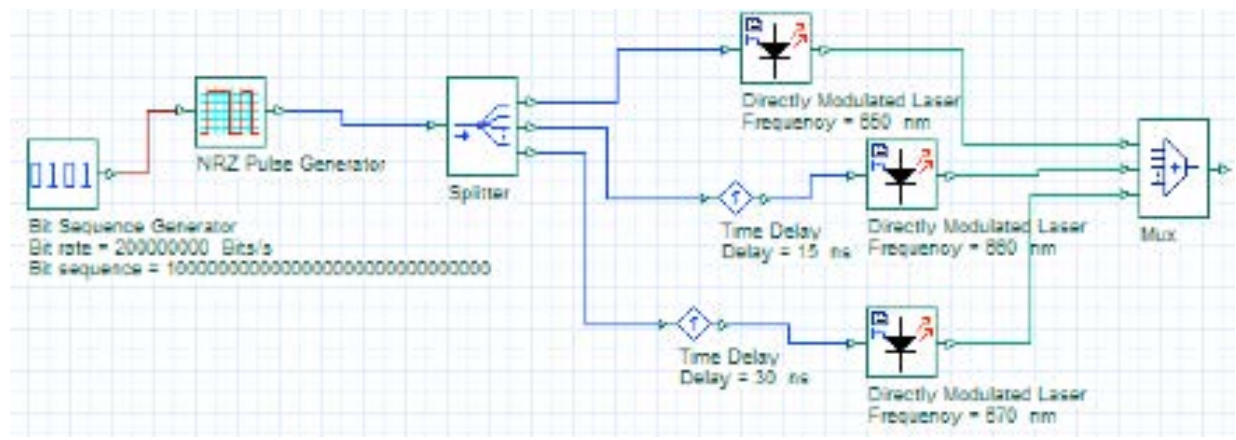

Figure 2. OptiSystem ${ }^{\mathrm{TM}}$ simulation set-up of the transmitter. Illustrated is the 2-D tuneable encoder with delaylines programmed for the CHPC sequence $X_{3}=(0,3,6)$ of $w=L=3, N=31$, and $\tau=5 \mathrm{~ns}$.

The reflected 2-D CHPC sequences (i.e., ToF signals) from the object and other transmitters will arrive at the receiver of the sensor. To distinguish the desired sequence from interfering sequences, the set-up of 2-D decoder is similar to that of the 2-D encoder (in Figure 2) but with reversed time delays. The decoder consists of a wavelength demultiplexer (Demux), a set of 3 APDs with tuneable delay-lines, and a $3 \times 1$ combiner. The Demux routes the arrival pulses to their corresponding wavelength paths (i.e., top, middle, and bottom paths for $\lambda_{0}=650$, $\lambda_{1}=660$, and $\lambda_{2}=670 \mathrm{~nm}$, respectively). The APDs converted optical pulses into electrical ones. Tuneable delaylines are used to reverse the time-delays of the pulses in accordance to the address signature of the decoder. For example, to decode $X_{3}=(0,3,6)$, the time delays on the $\lambda_{0}, \lambda_{1}$, and $\lambda_{2}$ wavelength paths are set to 30,15 , and $0 \mathrm{~ns}$, respectively. The delayed pulses from the three paths are multiplexed at the combiner to generate the correlation function. If the ToF signals consist of the desired sequence (i.e., $X_{3}$ ), an autocorrelation function with a high peak will be formed; otherwise, a low cross-correlation function will be generated. By threshold-detecting the autocorrelation peak, the desired ToF signal can be detected and the round-trip time can be measured.

The simulation set-up of the proposed 2-D OCDMA ToF LIDAR system consists of 16 encoders and one decoder of $X_{3}=(0,3,6)$. The CHPC sequences (out of a total of 31) created by the 16 encoders are $(0,2,4),(0,3,6)$, $(0,5,10),(0,6,12),(0,8,16),(0,10,20),(0,12,24),(0,14,28),(0,16,1),(0,19,7),(0,20,9),(0,22,13),(0,25,19)$, $(0,26,1),(0,28,25)$, and $(0,30,29)$. Tuneable delay-lines with different amounts of time delays are added to the outputs of these encoders to simulate the asynchronous nature of the system. A reflective filter is used to emulate the reflection of these 16 ToF signals to the decoder.

\section{2-D OCDMA TOF LIDAR SYSTEM SIMULATION RESULTS}

The simulation result of the proposed OCDMA LIDAR system with the 2-D CHPCs of $w=L=3$ and $N=31$ is shown in Figure 3a, which contains the accumulated correlation function of the 16 CHPC sequences at the output of the 2-D decoder of $X_{3}$. There are in total $16 \times 3=48$ pulses in the correlation function per period. Among them, only three pulses come from the desired sequence $X_{3}$ and will add atop at the decoder to give an autocorrelation peak of 3 units in height. The other 45 pulses, which are evenly spread out over one period in this simulation example, give the cross-correlation function of up to 2 units in height. Figure 3b shows that the simulation result of the 1-D OOC of $w=3$ and $N=31$. The simulation uses the OOC sequence $(0,3,15)$ as the desired sequence and 
$(0,1,7),(0,2,11),(0,4,14)$, and $(0,5,13)$ as the interfering sequences. Because each sequence contains three pulses (of same wavelength), a total of 15 pulses per period will arrive at the decoder of $(0,3,15)$. These 15 pulses are detected by an APD and then split by a $1 \times 3$ splitter into three paths, and each path contains the same 15 pulses [6], [7]. These pulses are time-delayed by the delay-lines on the three paths and recombined at a $3 \times 1$ combiner to give the accumulated correlation function of 45 pulses. Among them, only the three pulses coming from the desired sequence $(0,3,15)$ will add atop to give an autocorrelation peak. Using only one wavelength in the 1-D OOC, the simulation example in Figure 3b shows that three chips in the cross-correlation function are as high as the autocorrelation peak, thus creating false detection and range ambiguity and, in turn, ToF measurement errors. However, the 2-D CHPCs do not have these problems even with 16 sequences in use simultaneously.
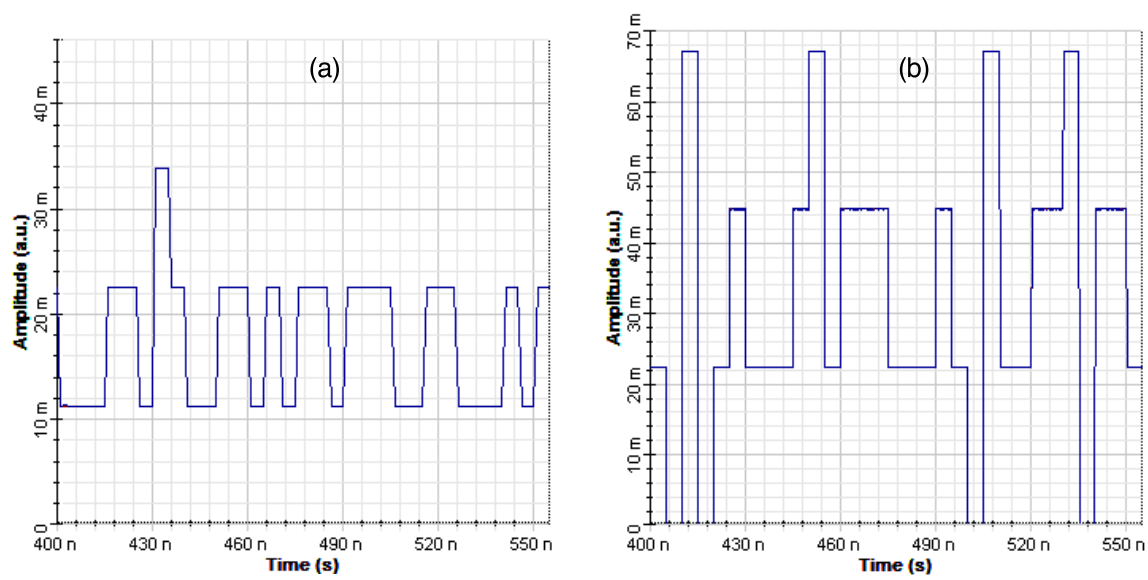

Figure 3. OCDMA ToF LIDAR system simulation results with a) 16 2-D CHPC and b) 5 1-D OOC sequences.

\section{CONCLUSIONS}

The 2-D OCDMA modulation in an automotive ToF LIDAR system was studied. The restrictions and regulations that governed the design of the proposed systems were presented. The 2-D modulation technique and hardware were implemented and validated with OptiSystem ${ }^{\mathrm{TM}}$ simulation. The interference-robustness studies showed that the proposed 2-D OCDMA automotive ToF LIDAR system was more flexible in the choice of system/code parameters and could support many more sensors and simultaneous ToF measurements than the 1-D counterpart.

\section{ACKNOWLEDGEMENTS}

This work was supported in part by the Faulty Development and Research Grants of Hofstra University, in part by the Ministry of Science and Technology of Republic of China under Grant MOST 107-2221-E-005-016-MY3, and in part by the European Union's Horizon 2020 research and innovation program under the Marie SklodowskaCurie grant agreement No 734331.

\section{REFERENCES}

[1] G. Kim and Y. Park, "LIDAR pulse coding for high resolution range imaging at improved refresh rate," Optics Express, vol. 24, no. 21, pp. 23810-23828, Oct. 2016.

[2] T. Fersch, R. Weigel, and A. Koelpin, “A CDMA modulation technique for automotive time-of-flight LiDAR systems,” IEEE Sensors J., vol. 17, no. 11, pp. 3507-3516, Jun. 2017.

[3] G. Kim and Y. Park, "Suitable combination of direct intensity modulation and spreading sequence for LIDAR with pulse coding,” MDPI Sensors, vol. 18, no. 12, pp. 1-22, Dec. 2018.

[4] F. R. K. Chung, J. A. Salehi, and V. K. Wei, “Optical orthogonal codes: Design, analysis, and applications,” IEEE Trans. Info. Theory, vol. 35, no. 3, pp. 595-604, May. 1989.

[5] W. C. Kwong, P. A. Perrier, and P. R. Prucnal, "Performance comparison of asynchronous and synchronous code-division multiple-access techniques for fiber-optic local area networks,” IEEE Trans. Commun., vol. 39, no. 11, pp. 1625-1634, Nov. 1991.

[6] G.-C. Yang and W. C. Kwong, Prime Codes with Applications to CDMA Optical and Wireless Networks, Norwood, MA: Artech House, 2002.

[7] W. C. Kwong and G.-C. Yang, Optical Coding Theory with Prime, New York: CRC Press, 2013.

[8] G.-C. Yang and W. C. Kwong, "Performance comparison of multiwavelength CDMA and WDMA+CDMA for fiber-optic networks,” IEEE Trans. Commun., vol. 45, no. 11, pp. 1426-1434, Nov. 1997.

[9] W. C. Kwong, G.-C. Yang, V. Baby, C.-S. Brés, and P. R. Prucnal, "Multiple-wavelength optical orthogonal codes under prime-sequence permutations for optical CDMA,” IEEE Trans. Commun., vol. 53, no. 1, pp. 117-123, Jan. 2005. 